\title{
Avaliação da Fragilidade de Doentes Renais Crônicos em Tratamento de Hemodiálise
}

\author{
Grasiéle Costa Matos ${ }^{1}$, Rosangela Moraes de Campos², Paulo Ricardo Moreira ${ }^{3}$, Michele \\ Ferraz Figueró ${ }^{4}$, Graziela Valle Nicolodi ${ }^{5}$, Rodrigo de Rosso Krug ${ }^{6}$, Kalina Durigon Keller ${ }^{7}$
}

\begin{abstract}
RESUMO
Este estudo teve como objetivo avaliar a presença e o grau de fragilidade de doentes renais crônicos em tratamento de hemodiálise estratificado por sexo, idade, prática de atividade física e fisioterapia. A fragilidade foi avaliada por meio de três questionários, sendo eles Edmonton Frail Scale, Índice de Vulnerabilidade Clínico Funcional-20 (IVCF-20) e Survey of Health Ageing and Retirement in Europe (Share-Flx). Os dados foram analisados por intermédio de média, desvio padrão, percentual, teste " $\mathrm{t}$ " student, Qui-quadrado de Pearson, Análise de Variância (Anova) e análise de resíduos ajustados. A amostra foi composta de 94 pacientes em tratamento de hemodiálise, quando foi possível identificar a presença de fragilidade mediante os três questionários utilizados, com uma prevalência entre 60,6 a 86,2\%. A fragilidade apresentou correlação apenas com gênero e idade. Conclui-se que a amostra estudada apresentou fragilidade principalmente nos graus de moderada a severa, posto que quanto maior a idade maior a severidade da fragilidade. Sugere-se a realização de novos estudos a fim de identificar precocemente a síndrome de fragilidade, possibilitando intervenções preventivas em doentes renais crônicos.
\end{abstract}

Palavras-chave: Fragilidade. Insuficiência renal crônica. Diálise renal.

\section{FRAILTY EVALUATION OF CHRONIC RENAL PATIENTS IN HEMODIALYSIS TREATMENT}

\section{ABSTRACT}

This study was aimed to evaluate the presence and degree of frailty of patients with chronic renal disease undergoing hemodialysis treatment stratified by sex, age, physical activity and physiotherapy practice. Fragility was evaluated through three questionnaires: Edmonton Frail Scale, Functional Clinical Vulnerability Index-20 (IVCF-20) and Survey of Health Aging and Retirement in Europe (SHARE-FIx). Data were analyzed using mean, standard deviation, percent, student's t-test, Pearson's Chi-square, Variance Analysis (Anova) and adjusted residue analysis. The sample was composed of 94 patients on hemodialysis treatment. It was possible to identify the presence of fragility in the sample and it showed correlation with gender and age, and with other variables no relation was found. It was concluded that the studied sample presented fragility mainly in the degrees of moderate to severe, and the greater the age, the greater the severity of the fragility. It is suggested that new studies be carried out in order to identify early the fragility syndrome, allowing some preventive intervention and chronic kidney patients.

Keywords: Fragility. Chronic renal insufficiency. Renal dialysis.

RECEBIDO EM: 3/9/2019

MODIFICAÇÕES SOLICITADAS EM: 8/11/2019

ACEITO EM: 30/3/2020

\footnotetext{
Aluna do curso de Fisioterapia, Universidade de Cruz Alta, Rio Grande do Sul, Brasil. http://lattes.cnpq.br/9884014129407920. https://orcid.org/0000-00016097-3961. gramatos.14@gmail.com

2 Aluna do curso de Fisioterapia, Universidade de Cruz Alta, Rio Grande do Sul, Brasil. http://lattes.cnpq.br/0996545645027787. https://orcid.org/0000-00032283-7090. rosangela.moraesdecampos1@gmail.com

Professor do Programa de Pós-Graduação em Atenção Integral à Saúde, Universidade de Cruz Alta, Rio Grande do Sul, Brasil. http://lattes.cnpq. br/1190498732376651. https://orcid.org/0000-0002-3001-1988.prm.paulomoreira@unicruz.edu.br

${ }^{4}$ Bacharel em Física. http://lattes.cnpq.br/9243516212890149. https://orcid.org/0000-0002-6996-0048. mferrazfigueiro@gmail.com

${ }_{5}^{5}$ Fisioterapeuta, Universidade de Cruz Alta, Rio Grande do Sul, Brasil. http://lattes.cnpq.br/2459200224348513. https://orcid.org/0000-0003-0933-5563. granicolodi@unicruz.edu.br

${ }^{6}$ Professor do Programa de Pós-Graduação em Atenção Integral à Saúde, Universidade de Cruz Alta, Rio Grande do Sul, Brasil. http://lattes.cnpq. br/4452161709794540.https://orcid.org/0000-0002-6701-0751.granicolodi@unicruz.edu.br

Fisioterapeuta, Universidade de Cruz Alta, Rio Grande do Sul, Brasil. http://lattes.cnpq.br/3680645826681593. https://orcid.org/0000-0003-2015-232X. kkeller@unicruz.edu.br
} 


\section{INTRODUÇÃO}

Nos últimos anos tem sido observado um crescimento na incidência de doenças crônicas não transmissíveis, em especial da Doença Renal Crônica (DRC). Esta é caracterizada pela perda lenta, progressiva e irreversível das funções renais em razão da destruição dos néfrons, resultando na incapacidade do organismo em manter o equilíbrio metabólico e hidroeletrolítico (SESSO et al., 2017).

Apesar dos diversos métodos de tratamento desta doença, o mais utilizado ainda é a hemodiálise (HD) (SESSO et al., 2017; THOMÉ et al., 2019). A HD substitui, parcialmente, a função renal, reverte os sintomas urêmicos e preserva a vida de paciente com DRC, porém as alterações degenerativas prosseguem, como a piora da condição física, que é multifatorial e está relacionada à restrição promovida pelo tratamento hemodialítico, depressão e outras alterações psicológicas e comportamentais relacionadas a essa rotina (FREIRE et al., 2013).

Os indivíduos submetidos a este tipo de tratamento sofrem uma série de alterações e intercorrências, como dor, câimbras, náuseas, vômitos, dispneia e hipotensão/hipertensão, que não somente comprometem o aspecto físico, mas também psicológico (SESSO et al., 2017; THOMÉ et al., 2019).

Assim, tanto a doença quanto o tratamento podem gerar um impacto negativo (ANTUNES et al., 2013), repercutindo na redução da capacidade funcional, força muscular dos pacientes e na qualidade de vida dos doentes renais (MARCHESAN et al., 2016; FASSBINDER et al., 2015).

Este impacto negativo sobre a saúde dos doentes renais leva a crer que eles possam desenvolver uma síndrome clínica chamada fragilidade (MORLEY et al., 2013), que se caracteriza pela diminuição da força energética e performance, desencadeando um declínio de múltiplos sistemas fisiológicos e orgânicos que levam a um estado de maior vulnerabilidade e limitação da capacidade de manutenção da homeostase no indivíduo (MORLEY et al., 2013).

A fragilidade gera prejuízos práticos à vida do paciente e de sua família, tanto clínica quanto psicológica e social, pois é associada a um maior risco de consequências adversas, como vulnerabilidade, dependência, hospitalização e óbitos (MORLEY et al., 2013).

Acredita-se que a avaliação da fragilidade possibilita a identificação das características a ela relacionadas, possibilitando a proposição de intervenções para prevenir a ocorrência de desfechos relacionados à incapacidade funcional e ao surgimento de doenças. Além disso, aliada à fragilidade, a DRC aumenta o risco de mortalidade (LEME et al., 2019).

Desse modo, o presente estudo teve como objetivo avaliar a presença e o grau de fragilidade de doentes renais crônicos em tratamento de hemodiálise, estratificado por sexo, idade, prática de atividade física e fisioterapia.

\section{MATERIAIS E MÉTODOS}

Foi realizado um estudo do tipo observacional transversal, descritivo e analítico, com uma amostra de 94 pacientes com DRC, submetidos ao tratamento de HD em uma clínica renal da Região Noroeste do Estado do Rio Grande do Sul. Todos os indivíduos aceitaram participar voluntariamente da pesquisa e assinaram o Termo de Consentimento Livre e Esclarecido. O projeto seguiu as recomendações da Resolução no 466/2012 do Conselho Nacional de Saúde, sendo aprovado pelo Comitê de Ética da Universidade de Cruz Alta sob parecer no 2.547.940.

Na seleção da amostra foram utilizados como critérios de inclusão ter o diagnóstico de DRC e estar em tratamento de HD há mais de seis meses, sendo excluídos do estudo aqueles pacientes que não aceitaram responder os questionários.

Para a avaliação da fragilidade foram utilizados os seguintes instrumentos: Edmonton Frail Scale (EFS), Índice de Vulnerabilidade Clinico Funcional-20 (IVCF) e Share-FIx. Os questionários foram aplicados antes ou durante as sessões de hemodiálise, no período de março a junho de 2018 na própria clínica renal, por pesquisadores previamente treinados.

O instrumento EFS avalia nove domínios: estado geral de saúde, independência funcional, suporte social, uso de medicamentos, nutrição, humor, continência, desempenho funcional e cognição. Este instrumento permite, além de identificar a presença de fragilidade, classificá-la quanto ao seu grau de severidade entre leve, moderada e severa, e identificar a vulnerabilidade do indivíduo para tal (PERNA; FRANCIS; BOLOGNA, 2017). Este instrumento foi validado para idosos brasileiros (FABRÍCIO-WEHBE et al., 2009).

Já o Instrumento IVCF, criado e validado no Brasil, apresenta como resultado a presença ou não de fragilidade, avaliando nove domínios, contemplando aspectos multidimensionais da condição de saúde, sendo constituído por 20 questões distribuídas em seções, como idade, autopercepção da saúde, incapa- 
cidades funcionais, cognição, humor, mobilidade, comunicação e comorbidades múltiplas (MORAES et al., 2016).

O Share-Flx permite identificar a tendência de se tornar frágil, pois possui as categorias não frágil, pré-frágil e frágil, possibilitando uma análise diferenciada dos demais instrumentos utilizados, pois admite uma classificação específica por sexo (ROMERO-ORTUNO, 2013). Este instrumento ainda não é validado no Brasil.

Para a caracterização da amostra foi calculado o índice de massa corporal (IMC), classificando os participantes adultos em baixo peso grave $(<16 \mathrm{~kg} / \mathrm{m} 2)$, baixo peso moderado $(16,0-16,9)$, baixo peso leve $(17,0$ $18,4)$, normais (18 a 24$)$ sobrepeso $(25$ a 29,9$)$ e obeso $(\leq 30)$, e os idosos em baixo peso, normal e sobrepeso, considerando normal de 22 a $27 \mathrm{~kg} / \mathrm{m}^{2}$ (ABESO, 2016).

Para a análise dos dados adotou-se nível de significância de $\alpha=5 \%$. Na análise univariada foram calculadas as medidas descritivas (mínimo, máximo, média, mediana e desvio padrão) para as variáveis quantitati- vas, e para as variáveis qualitativas foram elaboradas as tabelas de frequência. $\mathrm{Na}$ análise bivariada a associação entre duas variáveis qualitativas foi estimada pelo teste Qui-Quadrado de Pearson, complementado pela Análise de Resíduos Ajustados Padronizados. $\mathrm{Na}$ comparação das médias da idade e do tempo de HD entre os grupos dos instrumentos EFS, IVCF e Share-Flx, foram utilizados o teste t de Student para amostras independentes ou a Análise de Variância (Anova) de um fator com teste post hoc de Tukey.

\section{RESULTADOS}

Como pode ser visualizado na Tabela 1, a amostra total foi dividida por sexo para uma melhor análise dos dados. É importante salientar que a média de idade e o tempo de HD de ambos os sexos foi similar, porém as mulheres apresentaram peso corporal e, consequentemente, IMC menor que o grupo masculino.

Após análise dos três instrumentos utilizados, foi possível identificar a presença de fragilidade na amostra em diferentes graus, principalmente de forma moderada a severa, no instrumento EFS, o que pode ser observado na Tabela 2.

Tabela 1 - Caracterização geral de pacientes em hemodiálise. Região Noroeste do Rio Grande do Sul, Brasil, 2018 (n=94)

\begin{tabular}{|c|c|c|c|c|}
\hline Variáveis & 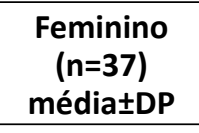 & $\begin{array}{c}\text { Masculino } \\
(n=57) \\
\text { média } \pm D P\end{array}$ & Total & $\mathbf{p}$ \\
\hline Idade & $57,27 \pm 16,62$ & $60,88 \pm 13,71$ & $59,46 \pm 14,94$ & 0,255 \\
\hline Peso & $59,88 \pm 15,02$ & $76,65 \pm 15,39$ & $70,09 \pm 17,25$ & 0,001 \\
\hline IMC & $23,25 \pm 5,03$ & $26,12 \pm 4,64$ & $25,00 \pm 4,98$ & 0,006 \\
\hline Tempo de hemodiálise (meses) & $67,49 \pm 61,50$ & $58,33 \pm 61,06$ & $62,01 \pm 61,45$ & 0,486 \\
\hline
\end{tabular}

Legenda: IMC = Índice de massa corporal; DP = Desvio padrão; teste t de student para amostras independentes com nível de significância de $p \leq 0,05$.

Fonte: Elaborado pelos autores (2008)

Tabela 2 - Fragilidade em pacientes em hemodiálise. Região Noroeste do Rio Grande do Sul, Brasil, 2018 ( $n=94$ )

\begin{tabular}{cllcc}
\hline Questionários & Classificação & Frequência & $\%$ \\
\hline \multirow{3}{*}{ Edmonton } & Não apresenta Fragilidade & 27 & 28,7 \\
& Aparentemente Vulnerável & 10 & 10,6 \\
& Fragilidade Leve & 16 & 17,0 \\
& & Fragilidade Moderada & 20 & 21,3 \\
& Fragilidade Severa & 21 & 22,3 \\
\hline \multirow{2}{*}{ IVCF } & Sem Fragilidade & 13 & 13,8 \\
& & Com Fragilidade & 81 & 86,2 \\
\hline \multirow{2}{*}{ Share fix } & Mulheres & Sem Fragilidade & 1 & 2,7 \\
& & Pré-Fragilidade & 15 & 40,5 \\
& & Com Fragilidade & 21 & 56,8 \\
\cline { 3 - 5 } & Homens & Sem Fragilidade & 3 & 5,3 \\
& & Pré-Fragilidade & 26 & 45,6 \\
& & Com Fragilidade & 28 & 49,1 \\
\hline
\end{tabular}

Fonte: Elaborado pelos autores (2008) 
É importante observar que, apesar das diferenças entre os questionários, relativas às categorias de classificação, os resultados foram semelhantes. Mediante o questionário EFS a fragilidade foi encontrada em $60,6 \%(n=57)$ da amostra, e no questionário IVCF foi possível verificar uma prevalência de $86,2 \%(n=81)$ da fragilidade. Esta maior incidência pode ser em razão da ausência de subclassificações neste instrumento. Já no Share-FIx, somando os sexos, foi identificada uma prevalência de $52,12 \%(n=49)$ de fragilidade. Assim, a fragilidade foi encontrada na maioria da amostra nos três instrumentos utilizados, porém em diferentes percentuais, o que deve ser relativo às diversas subclassificações de cada um.

Ao realizar o teste Qui-quadrado de Pearson, com nível de significância de $\alpha=5 \%$, entre os instrumentos utilizados foi identificada uma associação significativa entre os mesmos [EFS x IVCF $(p=0,001)$, com resíduos ajustados padronizados estatisticamente significativos (>|1,96|); para EFS x Share-Flx encontrou-se uma relação entre os homens ( $p=0,001)$, e para as muIheres obteve-se $p=0,005$, com resíduos ajustados padronizados estatisticamente significativos $(>|1,96|)]$, o que nos permite afirmar que os resultados para os instrumentos são equivalentes.

Para se verificar a associação entre fragilidade e gênero, foi utilizado o questionário Share-Flx, em razão da sua classificação da fragilidade por gênero.
Foi possível observar a prevalência de fragilidade em ambos os gêneros, o que pode ser visualizado na Tabela 2 .

Ao correlacionar a realização de fisioterapia ou outra atividade física regular com a presença de fragilidade, não foi encontrada diferença significativa relativa a quem não pratica alguma dessas atividades. Ou seja, independentemente de o indivíduo realizar fisioterapia ou praticar atividades físicas, ele pode apresentar um estado de fragilidade. Isto pode ser observado na Tabela 3 a seguir.

Mediante análise de variância foi possível verificar que, comparando com os três instrumentos utilizados - Instrumento escala de EFS ( $p=0,303)$; instrumento IVCF $(p=0,121)$ e instrumento Share-FIx para feminino $(p=0,779)$ e para masculino $(p=0,924)-$, o tempo de HD não teve correlação com a fragilidade.

Na Tabela 4 pode ser observada a associação entre a idade dos participantes e a fragilidade presente na amostra. Por meio do teste Anova e do teste post hoc de Tukey ( $\alpha=5 \%$ ), foi possível observar uma correlação significativa entre as médias de idade para as cinco subclassificações de fragilidade no questionário EFS. Assim, quanto mais avançada a idade mais severa é a fragilidade.

Tabela 3 - Correlação da fragilidade com tratamento de fisioterapia e outra atividade física por meio do Questionário de Edmonton de pacientes em hemodiálise. Região Noroeste do Rio Grande do Sul, Brasil, 2018 ( $n=94$ )

\begin{tabular}{lccccc}
\hline & $\begin{array}{c}\text { Não apresenta } \\
\text { fragilidade }\end{array}$ & $\begin{array}{c}\text { Aparentemente } \\
\text { vulnerável }\end{array}$ & Fragilidade leve & $\begin{array}{c}\text { Fragilidade } \\
\text { moderada }\end{array}$ & $\begin{array}{c}\text { Fragilidade } \\
\text { severa }\end{array}$ \\
\cline { 2 - 6 } Variáveis & & $\mathrm{n}(\%)$ & $\mathrm{p}$ & 0,781 \\
\hline $\begin{array}{l}\text { Fisioterapia } \\
\text { Sim }\end{array}$ & $4(4,3)$ & $2(2,1)$ & $5(5,3)$ & $5(5,3)$ & $5(5,3)$ \\
Não & $23(24,5)$ & $8(8,5)$ & $11(11,7)$ & $15(16,0)$ & $16(17,0)$ \\
Outra AF & $9(9,6)$ & $2(2,1)$ & $6(6,4)$ & $5(5,3)$ & $7(7,4)$ \\
$\quad$ Sim & $18(19 \%)$ & $8(8,5)$ & $10(10,6)$ & $15(16,0)$ & $14(14,9)$ \\
Não & & & & & 0,854 \\
\hline
\end{tabular}

Fonte: Elaborado pelos autores (2008)

Tabela 4 - Comparação das médias de idade com a fragilidade por intermédio do questionário de Edmonton de pacientes em hemodiálise. Região Noroeste do Rio Grande do Sul, Brasil, 2018 (n=94)

\begin{tabular}{lllll}
\hline Fragilidade & $\mathrm{n}$ & média & $\mathrm{DP}$ & $\mathrm{P}$ \\
\hline Não apresenta fragilidade & 27 & 47,30 & 12,84 & \\
Aparentemente vulnerável & 10 & 58,00 & 13,78 & \\
Fragilidade leve & 16 & 62,44 & 14,76 & 0,001 \\
Fragilidade moderada & 20 & 65,30 & 13,12 & \\
Fragilidade severa & 19 & 67,95 & 9,94 & \\
\hline
\end{tabular}

Legenda: Realizada Anova para as médias de idade para os 5 grupos ( $\alpha=5 \%$ ) e Post hoc de Tukey ( $\alpha=5 \%$ ) entre os grupos. DP= Desvio Padrão. 


\section{DISCUSSÃO}

Neste estudo foi observada a prevalência de fragilidade em doentes renais crônicos, variando entre $60,6 \%$ a $86,2 \%$ do total da amostra, conforme o questionário analisado, principalmente em graus moderado a grave. Os doentes renais crônicos submetidos a tratamento de HD acabam tendendo a uma capacidade funcional menor em relação aos indivíduos normais, o que causa repercussões em diversos sistemas do corpo, deixando-os de fato mais fragilizados, o que influencia diretamente nos índices de morbidade e mortalidade (OLIVEIRA; VIEIRA; BÜNDCHEN, 2018). Assim, os pacientes com DRC têm duas vezes mais chances de desenvolverem a fragilidade que indivíduos com função renal normal (MANSUR; DAMASCENO; BASTOS, 2012).

Segundo a literatura científica, a fragilidade é mais prevalente em homens doentes renais (BIAVO et al., 2012; ORLANDI et al., 2012); na população saudável é mais prevalente em mulheres (ARAÚJO; CARVALHO; MENESES, 2016), contudo, neste estudo a fragilidade foi observada em ambos os sexos, e, ainda, quando avaliados em separado, as mulheres apresentaram um percentual maior de fragilidade $(56,8 \%)$ relativo aos homens $(49,1 \%)$. Foi encontrada uma associação entre os instrumentos de fragilidade mesmo quando correlacionados, equivalendo seus resultados. Este dado é fundamental, pois cada questionário tem suas particularidades de classificação como a separação por sexo.

Apesar de a prática de atividades físicas preservarem a independência nas atividades de vida diária (VIRTUOSO JÚNIOR et al., 2016) e contribuírem para a redução e o controle de fatores de risco para doenças cardiovasculares (BARBOSA et al., 2018), isto não foi observado nos doentes renais estudados. Ao analisarmos a relação da prática de fisioterapia ou outra atividade física com a presença de fragilidade, não foi encontrada relevância significativa, sugerindo que nesta amostra de indivíduos com deficiência na função renal, mesmo estando ativos fisicamente, apresentaram estado de fragilidade. Cabe ressaltar, porém, que não foi avaliada a frequência e a intensidade destes treinamentos físicos questionados.

Em um estudo que investigou a fragilidade e os fatores associados a essa condição, considerando o nível de atividade física dos idosos, foi observada uma moderada prevalência $(37,4 \%)$ de pré-fragilidade, mesmo em indivíduos praticantes de atividade física (LENARDT et al., 2013).
No presente estudo foram avaliados indivíduos com tempo de exposição à HD bem distintos, desde 2 a 30 anos, com o intuito de verificar se o tempo de tratamento influencia no surgimento da fragilidade. Este aspecto teve um resultado interessante, mostrando que o tempo de tratamento não tem relação com o surgimento da fragilidade. Isto é confirmado pela literatura, que afirma que o que mais influencia na fragilidade, sendo preditor de mortalidade, é a idade avançada ao iniciar a HD, além dos níveis baixo de hemoglobina e albumina (TEIXEIRA et al., 2015).

Neste estudo foi possível perceber que quanto maior a idade mais severa a fragilidade. Paralelo ao avanço da expectativa de vida, ocorre uma maior incidência de agravos à saúde, dentre os quais destacam-se o diabetes mellitus e a hipertensão arterial sistêmi$\mathrm{ca}$, que contribuem para o aumento da prevalência da DRC (PEREIRA et al., 2014). A presença de DRC, como visto neste estudo, também influencia na prevalência de fragilidade, formando um ciclo entre doença, idade avançada e fragilidade.

Tendo em vista a alta prevalência e o grau de severidade de fragilidade encontrados nos doentes renais crônicos em tratamento de hemodiálise estudados neste estudo, vê-se a importância desta variável no tratamento hemodialítico, considerando a grande influência que a fragilidade tem sobre a saúde desta população. Neste sentido, avaliar a fragilidade de pacientes em HD deve ser uma constante em clínicas renais. Outro ponto positivo do estudo é que a fragilidade foi avaliada por meio de três questionários (Edmonton Frail Scale, IVCF-20 e Share-FIx), posto que todos mostraram alta prevalência e severidade da variável pesquisada.

Como limitação do estudo pode-se destacar, principalmente, a falta de avaliação da frequência da participação dos pacientes em hemodiálise na prática de fisioterapia e atividade física, tendo em vista que este comportamento ativo pode influenciar na redução da fragilidade.

Sugere-se que a fragilidade seja acompanhada de forma longitudinal nestes pacientes, para serem verificados outros fatores que podem interferir neste acometimento em pacientes renais.

\section{CONCLUSÃO}

Conclui-se que a fragilidade estava presente na maioria (mais de $70 \%$ ) dos doentes renais avaliados, sendo os graus moderado e severo os mais prevalentes. Além disto, a idade avançada foi um fator significativo para a presença de fragilidade, diferente do 
sexo e tempo de HD, que não tiveram associação para tal. Sugere-se que sejam realizados mais estudos nessa área, em razão do aumento da incidência da DRC e as comorbidades associadas a ela.

\section{REFERÊNCIAS}

ANTUNES, M. et al. Quando uma máquina mantém a vida: o itinerário do idoso renal crônico em hemodiálise. Revista Contexto \& Saúde, ljuí: Editora Unijuí, v. 11, n. 20, p. 1.2831.286, 2013.

ARAÚJO, L. F.; CARVALHO, C. M. G.; MENESES, C. C. C. F. Representações sociais sobre fragilidade: concepções de idosos na atenção básica de saúde. Estudos Interdisciplinares sobre o Envelhecimento, v. 21, n. 2, 2016.

ABESO. Associação Brasileira para o Estudo da Obesidade e da Síndrome Metabólica. Diretrizes Brasileiras de Obesidade 2016. 4. ed. São Paulo: Abeso, 2016.

BARBOSA, J. P. dos A. S. et al. Relação entre atividade física, aptidão física e risco cardiovascular: estudo em Muzambinho, Minas Gerais. Revista Brasileira de Medicina do Esporte, v. 24, n. 1, p. 73-77, 2018.

BIAVO, B. M. et al. Aspectos nutricionais e epidemiológicos de pacientes com doença renal crônica submetidos a tratamento hemodialítico no Brasil. Jornal Brasileiro de Nefrologia, v. 2, n. 34, p. 206-215, 2012.

FABRÍCIO-WEHBE, S. C. C. et al. Adaptação cultural e validade da Edmonton Frail Scale-EFS em uma amostra de idosos brasileiros. Revista Latino-Americana de Enfermagem, v. 17, n. 6, 2009.

FASSBINDER, T. R. C. et al. Capacidade funcional e qualidade de vida de pacientes com doença renal crônica pré-dialítica e em hemodiálise - um estudo transversal. Jornal Brasileiro de Nefrologia, n. 1, v. 37, 2015.

FREIRE, A. P. C. F. et al. Aplicação de exercício isotônico durante a hemodiálise melhora a eficiência diálitica. Fisioterapia em Movimento, v. 26, n. 1, p. 167-174, 2013.

LEME, D. E. da C. et al. Estudo do impacto da fragilidade, multimorbidade e incapacidade funcional na sobrevida de idosos ambulatoriais. Ciência e Saúde Coletiva, v. 24, n. 1, p. 137-146, 2019.

LENARDT, M. H. et al. Atividade física de idosos e fatores associados à pré-fragilidade. Acta Paulista de Enfermagem, v. 26, n. 3, p. 269-275, 2013.

MANSUR, H. N.; DAMASCENO, V. O.; BASTOS, M. G. Prevalência da fragilidade entre os pacientes com doença renal crônica em tratamento conservador e em diálise. Jornal Brasileiro de Nefrologia, v. 34, n. 2, p. 153-160, 2012.

MARCHESAN, M. et al. Physical exercise modifies the functional capacity of elderly patients on hemodialysis. Fisioterapia em Movimento, v. 29, n. 2, p. 351-359, 2016.

MORAES, E. M. et al. Índice de Vulnerabilidade Clínico Funcional-20 (IVCF-20): reconhecimento rápido do idoso frágil. Revista de Saúde Pública, São Paulo, v. 50, dez. 2016.
MORLEY, J. E. et al. Frailty consensus: a call to action. Journal of the American Medical Directors Association, v. 14, n. 6, p. 392-397, 2013.

OLIVEIRA, A. C. F.; VIEIRA, D. S. R.; BÜNDCHEN, D. C. Nível de atividade física e capacidade funcional de pacientes com doença renal crônica pré-dialítica e em hemodiálise. Fisioterapia e Pesquisa, v. 25, n. 3, p. 323-329, 2018.

ORLANDI, F. S. et al. Avaliação do nível de esperança de vida de idosos renais crônicos em hemodiálise. Revista da Escola de Enfermagem da USP, v. 46, n. 4, p. 500-505, 2012.

PEREIRA, E. R. et al. Análise das principais complicações durante a terapia hemodialítica em pacientes com insuficiência renal crônica. Revista de Enfermagem do Centro Oeste Mineiro, v. 4, n. 2, p. 1.123-1.134, 2014.

PERNA, S. FRANCIS, M. D.; BOLOGNA, C. Performance of Edmonton Frail Scale on frailty assessment: its association with multi-dimensional geriatric conditions assessed with specific screening tools. BMC Geriatrics, v. 17, n. 1, p. 1-10, 2017.

ROMERO-ORTUNO, R. The Frailty Instrument for primary care of the Survey of Health, Ageing and Retirement in Europe predicts mortality similarly to a frailty index based on comprehensive geriatric assessment. Geriatric and Gerontology International, v. 13, n. 2, p. 497-504, 2013.

SESSO, R. et al. Report of the Brazilian dialysis census. Jornal Brasileiro de Nefrologia, v. 39, n. 3, p. 261-266, 2017.

TEIXEIRA, F. I. R. et al. Sobrevida de pacientes em hemodiálise em um hospital universitário. Jornal Brasileiro de Nefrologia, v. 37, n. 1, p. 64-71, 2015.

THOMÉ, F. S. et al. Inquérito brasileiro de diálise crônica 2017. Jornal Brasileiro de Nefrologia, v. 41, n. 2, 208-214, 2019.

VIRTUOSO-JÚNIOR, J. S. et al. Fatores associados à incapacidade funcional em idosos brasileiros. Revista Andaluza de Medicina del Deporte, p. 1-7, 2016. Disponível em: http:// dx.doi.org/10.1016/j.ramd.2016.05. 